\title{
THE SEMIDIRECTLY CLOSED PSEUDOVARIETY GENERATED BY APERIODIC BRANDT SEMIGROUPS
}

\author{
M. LURDES TEIXEIRA
}

\begin{abstract}
This paper presents a study of the semidirectly closed pseudovariety generated by the aperiodic Brandt semigroup $B_{2}$, denoted $\mathbf{V}^{*}\left(B_{2}\right)$. We construct a basis of pseudoidentities for the semidirect powers of the pseudovariety generated by $B_{2}$ which leads to the main result, which states that $\mathbf{V}^{*}\left(B_{2}\right)$ is decidable.

Independently, using some suggestions given by J. Almeida in his book "Finite Semigroups and Universal Algebra", we constructed an algorithm to solve the membership problem in $\mathbf{V}^{*}\left(B_{2}\right)$.
\end{abstract}

\section{INTRODUCTION}

Recall that a pseudovariety of semigroups is a class of finite semigroups closed under taking divisors and finite direct products. The semidirect product $\mathbf{V} * \mathbf{W}$ of two pseudovarieties of semigroups is the pseudovariety generated by all semidirect products of semigroups of $\mathbf{V}$ by semigroups of $\mathbf{W}$ [7]. This definition gives an operation on the set of pseudovarieties that is associative and whose idempotents are precisely the semidirectly closed (abbreviated s.c.) pseudovarieties. In the theory of finite semigroups, the semidirect product is a construction that has drawn the attention of many authors. The study of the decidability of semidirect product pseudovarieties assumes particular relevance, since decidability of pseudovarieties is not preserved by the semidirect product operation [11].

The pseudovariety $\mathbf{V}^{*}\left(B_{2}\right)$ is the smallest s.c. pseudovariety that contains $B_{2}$, where $B_{2}$ is the aperiodic Brandt semigroup with five elements. In the lattice of s.c. pseudovarieties of aperiodic semigroups, $\mathbf{V}^{*}\left(B_{2}\right)$ covers $\mathbf{R}$ (the pseudovariety of all semigroups whose Green

Date: May 1999, revised April 2000.

1991 Mathematics Subject Classification. 20M07, $20 \mathrm{M} 05$.

Key words and phrases. Semigroup, semigrupoid, graph, pseudovariety, implicit operation, pseudoidentity, decidability, semidirect product, semidirectly closed, algorithm.

This work was supported by Centro de Matemática da Universidade do Minho and by project Praxis/2/2.1/MAT/63/94. 
relation $\mathcal{R}$ is trivial) and $\mathbf{V}^{*}\left(B_{2}\right)$ is a subpseudovariety of $\mathbf{E R} \cap \mathbf{L R}$ (the intersection of the pseudovariety of all semigroups whose subsemigroups generated by idempotents are members of $\mathbf{R}$ with the pseudovariety of all semigroups whose local subsemigroups are in $\mathbf{R}$ ) [1]. In [13] has been proved that $\mathbf{V}^{*}\left(B_{2}\right) \neq \mathbf{E R} \cap \mathbf{L R}$ and that the interval $\left[\mathbf{V}^{*}\left(B_{2}\right), \mathbf{E R} \cap \mathbf{L R}\right]$ contains a chain, for the inclusion relation, of s.c. pseudovarieties isomorphic to the chain of real numbers.

In this work we are going to study $\mathbf{V}^{*}\left(B_{2}\right)$ in order to prove its decidability. The pseudovariety $\mathbf{V}^{*}\left(B_{2}\right)$ is the direct union of the semidirect powers of $\mathbf{V}\left(B_{2}\right)$ (the pseudovariety generated by $B_{2}$ ) whose bases of pseudoidentities are constructed, recursively, using theorems [6, theorem 5.3] and [4, theorem 5.9]. Such bases are not effective, but they are used to obtain a decision criterion for the membership problem for $\mathbf{V}^{*}\left(B_{2}\right)$.

In [1], J. Almeida gives a suggestion to study the decidability of the membership problem of $\mathbf{V}^{*}\left(B_{2}\right)$. This arguments and the knowledge of the validity of some pseudoidentities in $\mathbf{V}^{*}\left(B_{2}\right)$ lead to an independent proof of decidability of $\mathbf{V}^{*}\left(B_{2}\right)$, because they allow us to construct an algorithm of polynomial complexity to solve the membership problem for $\mathbf{V}^{*}\left(B_{2}\right)$.

The basic results that we are going to use, about pseudovarieties of semigroups and of semigroupoids that contain $B_{2}$, are proved in $[4,13]$ and are based on the theory of finite semigroupoids and some previous results obtained by B. Tilson in [14] and by N. Reilly in [9].

\section{PRELIMINARIES}

For general background and terminology from the classical theory of semigroups, profinite semigroups and semidirect products, the reader is referred to $[8,1,5,14,6]$.

In this paper we are not going to consider empty algebras. We are going to consider topological algebras (total or partial) and, in particular, finite algebras are supposed to be discrete topological spaces.

In this section we are going to present a brief description of the most relevant basic concepts and propositions in order to make it easier to read the following sections.

\subsection{Free profinite semigroups.}

For a set $X$ endowed with a topology, we say that a semigroup $S$ is $X$-generated if there is a continuous function, from $X$ to $S$, such that $S$ is the smallest closed semigroup that contains the image of $X$. 
For a class $V$ of finite semigroups, we say that $S$ is pro- $V$ if it is the projective limit of semigroups of $V$.

For a pseudovariety $\mathbf{V}$ and a set $X$, the $X$-generated elements of $\mathbf{V}$ form a directed system and the respective projective limit will be denoted $\bar{\Omega}_{X} \mathbf{V}$. The semigroup $\bar{\Omega}_{X} \mathbf{V}$ is the free semigroup over $X$ for the class of all pro- $\mathbf{V}$ semigroups, which means that, for every pro- $\mathbf{V}$ semigroup $S$ and continuous function $\varphi: X \longrightarrow S$, there is a unique continuous morphism $\bar{\varphi}: \bar{\Omega}_{X} \mathbf{V} \longrightarrow S$ extending $\varphi$. The free semigroup over $X$ for the variety generated by $\mathbf{V}$ is denoted $\Omega_{X} \mathbf{V}$ and is a dense subsemigroup of $\bar{\Omega}_{X} \mathbf{V}$.

The elements of $\bar{\Omega}_{X} \mathbf{V}$ are usually called implicit operations, since $\bar{\Omega}_{X} \mathbf{V}$ is isomorphic to the semigroup of implicit operations on $\mathbf{V}$ over $X$. If $X$ is an alphabet that contains $x$, then the implicit operation represented by $x^{\omega}$ is such that, for each finite semigroup $S$ and each continuous function $\varphi: X \longrightarrow S$, if $\varphi(x)=s$ then $\bar{\varphi}\left(x^{\omega}\right)=s^{n}$, the power of $s$ that is idempotent.

Given a profinite set $X$ and a pseudovariety $\mathbf{V}$ (usually $\mathbf{V}=\mathbf{S}$, the pseudovariety of all finite semigroups), a pseudoidentity over $\mathbf{V}$ is a formal equality $\pi=\rho$ where $\pi, \rho \in \bar{\Omega}_{X} \mathbf{V}$. A semigroup $S \in \mathbf{V}$ satisfies the pseudoidentity $\pi=\rho$, or equivalently, $\pi=\rho$ holds in $S$, if for every continuous function $\varphi: X \longrightarrow S, \bar{\varphi}(\pi)=\bar{\varphi}(\rho)$ and we write $S=\pi=\rho$. For a subclass $C$ of $\mathbf{V}$ we say that $C$ satisfies the pseudoidentity $\pi=\rho$ if all elements of $C$ satisfy $\pi=\rho$, and we write $C \models \pi=\rho$. For a set $\Sigma$ of pseudoidentities, $\llbracket \Sigma \rrbracket$ denotes the class of all finite semigroups in which all pseudoidentities of $\Sigma$ hold. In [10] J. Reiterman proves that the set of pseudovarieties and the set of classes of the form $\llbracket \Sigma \rrbracket$ are equal, where $\Sigma$ is a set of pseudoidentities over finite sets. If $\mathbf{V}=\llbracket \Sigma \rrbracket$ then $\Sigma$ is said to be a basis of pseudoidentities of $\mathbf{V}$.

Given $\pi \in \bar{\Omega}_{X} \mathbf{S}, \rho \in \bar{\Omega}_{X} \mathbf{S}$ is a factor of $\pi$ if there are $\pi_{1}, \pi_{2} \in\left(\bar{\Omega}_{X} \mathbf{S}\right)^{1}$ such that $\pi=\pi_{1} \rho \pi_{2}$. If $\rho \in X$ and $\pi_{1}=1$ ( or $\pi_{2}=1$ ) then $\rho$ is denoted by $i(\pi)$ (or $t(\pi)$, respectively). By $C(\pi)$ we denote the content of $\pi$ which is the subset of $X$ consisting of all elements of $X$ that are factors of $\pi$.

\subsection{Free profinite semigroupoids and semidirect products.}

By a (directed) graph $G$ we mean a partial algebra with a support set $V(G) \stackrel{\circ}{\cup} E(G)$ with two sorts of elements, called vertices and edges respectively, and two binary operations: $\alpha: E(G) \longrightarrow V(G)$ and $\omega: E(G) \longrightarrow V(G)$. For $c, d \in V(G), G(c, d)$ is the set of edges $s$ of $G$ such that $\alpha(s)=c$ and $\omega(s)=d$. Two edges $s_{1}$ and $s_{2}$ are said to be consecutive if $\omega\left(s_{1}\right)=\alpha\left(s_{2}\right)$, and are said to be coterminal if 
$\alpha\left(s_{1}\right)=\alpha\left(s_{2}\right)$ and $\omega\left(s_{1}\right)=\omega\left(s_{2}\right)$. A finite sequence of consecutive edges of $G$ is a path of $G$. We define the graph $G^{+}$as the graph such that $V\left(G^{+}\right)=V(G), E\left(G^{+}\right)$is the set of all paths in $G$ and, for $v_{1} v_{2} \ldots v_{n}$ a path in $G, \alpha\left(v_{1} v_{2} \ldots v_{n}\right)=\alpha\left(v_{1}\right)$ and $\omega\left(v_{1} v_{2} \ldots v_{n}\right)=\omega\left(v_{n}\right)$. The graph $G$ is said to be connected if, for every pair of vertices $(p, q)$, there is a finite sequence of vertices $v_{0}, v_{1}, \ldots, v_{n}$ such that $v_{0}=p, v_{n}=q$ and $G\left(v_{i}, v_{i+1}\right) \cup G\left(v_{i+1}, v_{i}\right) \neq \emptyset$, for $i=1, \ldots, n-1$, and $G$ is said to be strongly connected if, for every pair of vertices $(p, q), G^{+}(p, q) \neq \emptyset$.

A semigroupoid $S$ is a graph with an associative partial operation, called composition, whose domain is $\left\{(s, t) \in E(S)^{2}: \alpha(t)=\omega(s)\right\}$, and such that given $(s, t)$ in the domain their composition is an edge called $s t$ which lies in $S(\alpha(s), \omega(t))$. A category $S$ is a semigroupoid that has an identity element at each vertex, which means that, for every $v \in V(S)$, there is $1_{v} \in S(v, v)$ such that, whenever the compositions are defined in $S, s 1_{v}=s$ and $1_{v} s=s$. For a semigroupoid $S$, we denote by $S^{c}$ the smallest category that contains $S$. Semigroups can be interpreted as semigroupoids with only one vertex.

For graphs, semigroupoids and categories, morphisms are defined as functions between graphs, semigroupoids and categories, respectively, respecting sorts and operations. A morphism between the semigroupoids $S$ and $T, \psi: S \longrightarrow T$, is said to be:

- faithful if, for every $c, d \in V(S)$, the restriction $\psi_{\mid S(c, d)}$ is injective;

- a quotient morphism if $\psi$ is surjective and the restriction $\psi_{\mid V(S)}$ is injective;

- an isomorphism if $\psi$ is bijective.

A semigroupoid $S$ is a quotient of a semigroupoid $T$ if there is a quotient morphism $\psi: T \longrightarrow S$. We say that a semigroupoid $S$ divides a semigroupoid $T$, and we write $S \prec T$, if $S$ is a quotient of a semigroupoid $E$ for which exists a faithful morphism $\beta: E \longrightarrow T$.

A variety (pseudovariety) of semigroupoids $\mathbf{V}$ is a class of (finite) semigroupoids containing a semigroupoid with just one vertex and one edge which is closed under taking (finite) divisors and (finitary) products and coproducts. For a pseudovariety $\mathbf{W}$ of semigroups we define $g \mathbf{W}$ to be the pseudovariety of semigroupoids generated by the elements of $\mathbf{W}$.

For semigroupoids we can construct a theory similar to the semigroup case, by defining free profinite semigroupoids. As in the case of semigroups, the projective limit of the $G$-generated semigroupoids of $\mathbf{V}, \bar{\Omega}_{G} \mathbf{V}$, is the free semigroupoid generated by the graph $G$ in the class of all pro- $\mathbf{V}$ semigroupoids. The concept of implicit operation of semigroupoids is obtained by extension of the concept of implicit operation 
of semigroups, in such a way that the set of all implicit operations assumes the structure of a semigroupoid that is isomorphic to $\bar{\Omega}_{G} \mathbf{V}$.

A pseudoidentity is a formal equality between two coterminal edges $\pi$ and $\rho$ of $\bar{\Omega}_{G} \mathbf{V}$, denoted $(\pi=\rho, G)$. In case $G$ is finite and connected, we say that a semigroupoid $S \in \mathbf{V}$ satisfies a pseudoidentity $(\pi=\rho, G)$ (or that $(\pi=\rho, G)$ holds in $S)$ if $\bar{\varphi}(\pi)=\bar{\varphi}(\rho)$, for every graph morphism $\varphi: G \longrightarrow S$ and $\bar{\varphi}: \bar{\Omega}_{G} \mathbf{V} \longrightarrow S$ the unique continuous extension of $\varphi$. Usually we will consider pseudoidentities on Sd (the pseudovariety of all finite semigroupoids). An extension of Reiterman's Theorem is valid, which means that each pseudovariety of semigroupoids can be defined by a set of pseudoidentities over finite connected graphs.

By a labeling of a graph $G$ by $\bar{\Omega}_{X} \mathbf{S}$ we mean a pair $\left(\left(\pi_{q}\right)_{q \in V(G)}, \varepsilon\right)$ where $\left(\pi_{q}\right)_{q \in V(G)}$ is a family of elements of $\left(\bar{\Omega}_{X} \mathbf{S}\right)^{1}$ and $\varepsilon: E(G) \longrightarrow \bar{\Omega}_{X} \mathbf{S}$ is a map. Such labeling is compatible with a pseudovariety $\mathbf{V}$ if, for all $s \in E(G), \mathbf{V} \models\left(\pi_{\alpha(s)} \cdot \varepsilon(s)=\pi_{\omega(s)}\right)$. The process to construct a basis of pseudoidentities for pseudovarieties of semigroups of the form $\mathbf{V} * \mathbf{W}$ is stated in the following theorem.

Theorem 2.1. [6, theorem 5.3] Let $\mathbf{V}$ and $\mathbf{W}$ be pseudovarieties of semigroups. If $\Sigma$ is a pseudoidentity basis of $g \mathbf{V}$, then the set of the pseudoidentities of the form

$$
\pi_{\alpha(u)} \varepsilon(u)=\pi_{\alpha(v)} \varepsilon(v),
$$

where $(u=v, G) \in \Sigma, X$ is a finite set, $\left(\left(\pi_{q}\right)_{q \in V(G)}, \varepsilon^{\prime}\right)$ is a labeling of $G$ by $\bar{\Omega}_{X} \mathbf{S}$ compatible with $\mathbf{W}$ and $\varepsilon: \bar{\Omega}_{G} \mathbf{S d} \longrightarrow \bar{\Omega}_{X} \mathbf{S}$ is the continuous morphism of semigroupoids constant on vertices and defined on edges by: $\varepsilon(s)=\varepsilon^{\prime}(s)$, for every $s \in E(G)$, is a pseudoidentity basis for the pseudovariety of semigroups $\mathbf{V} * \mathbf{W}$.

\subsection{Some results about pseudovarieties which contain $B_{2}$.}

By definition, a Brandt semigroup is a completely 0-simple inverse semigroup. So a Brandt semigroup is isomorphic to a $I \times I$ Rees matrix semigroup over a 0 -group, $G^{0}$, with the identity matrix. Usually we denote by $B_{n}$ the aperiodic Brandt semigroup such that $\sharp I=n$. The set $\left\{x^{2}=x^{3}, x^{2} y^{2}=y^{2} x^{2}, x(y x)^{2}=x y x\right\}$ is an identity basis of the variety of semigroups generated by $B_{2}, V\left(B_{2}\right)$, [15]. The pseudovariety of semigroups generated by $B_{2}, \mathbf{V}\left(B_{2}\right)$, is equal to $V\left(B_{2}\right) \cap \mathbf{S}$ and so

$$
\mathbf{V}\left(B_{2}\right)=\llbracket x^{2}=x^{3}, x^{2} y^{2}=y^{2} x^{2}, x(y x)^{2}=x y x \rrbracket .
$$


A generalization of N. Reilly's solution of the word problem in free inverse semigroups to the profinite semigroups $\bar{\Omega}_{X} \mathbf{V}\left(B_{2}\right)$ was obtained in [4] and the result is presented in theorem 2.2 below. To understand the meaning of this theorem we need to introduce some notions and terminology.

For a set $X, X^{-1}=\left\{x^{-1}: x \in X\right\}$ is a disjoint copy of $X$. Given a finite alphabet $X$ and $\pi \in \bar{\Omega}_{X} \mathbf{S}, \delta_{\pi}$ is the equivalence relation over $C(\pi) \cup C(\pi)^{-1}$ generated by the set

$$
\left\{\left(x^{-1}, y\right): x, y \in C(\pi), x y \text { is a factor of } \pi\right\} .
$$

Given an alphabet $X$ and $\pi \in \bar{\Omega}_{X} \mathbf{S}$, we define $\mathcal{A}_{\pi}$ to be the graph:

- $V\left(\mathcal{A}_{\pi}\right)=\left(C(\pi) \cup C(\pi)^{-1}\right) / \delta_{\pi}$;

- for any $v_{1}, v_{2} \in V\left(\mathcal{A}_{\pi}\right)$,

$$
\mathcal{A}_{\pi}\left(v_{1}, v_{2}\right)=\left\{x \in C(\pi):[x]_{\delta_{\pi}}=v_{1} \text { and }\left[x^{-1}\right]_{\delta_{\pi}}=v_{2}\right\} \text {. }
$$

The vertices $[i(\pi)]_{\delta_{\pi}}$ and $\left[t(\pi)^{-1}\right]_{\delta_{\pi}}$ are called the initial vertex and the final vertex of $\mathcal{A}_{\pi}$, respectively.

Since the $\mathbf{V}\left(B_{2}\right)$ is finitely generated, $\Omega_{X} \mathbf{V}\left(B_{2}\right)=\bar{\Omega}_{X} \mathbf{V}\left(B_{2}\right)$ and, using [9, theorem 3.3], the proof of the next theorem is an obvious consequence of the fact that the function that associates to each implicit operation $\pi$ the relation $\delta_{\pi}$, is a continuous function.

Theorem 2.2. [4, theorem 5.6 ] Given a finite alphabet $X$ and $\pi_{1}, \pi_{2} \in \bar{\Omega}_{X} \mathbf{S}$, the pseudoidentity $\pi_{1}=\pi_{2}$ holds in $B_{2}$ if and only if:

1. $\mathcal{A}_{\pi_{1}}=\mathcal{A}_{\pi_{2}}$

2. $\left[i\left(\pi_{1}\right)\right]_{\delta_{\pi_{1}}}=\left[i\left(\pi_{2}\right)\right]_{\delta_{\pi_{2}}}$ and $\left[t\left(\pi_{1}\right)^{-1}\right]_{\delta_{\pi_{1}}}=\left[t\left(\pi_{2}\right)^{-1}\right]_{\delta_{\pi_{2}}}$.

It follows from the definition that, if $u$ is a word factor of $\pi$ then $u$ defines a path in $\mathcal{A}_{\pi}$ from $[i(u)]_{\delta_{\pi}}$ to $\left[(t(u))^{-1}\right]_{\delta_{\pi}}$.

In [3] it was proved that $E\left(\bar{\Omega}_{G} \mathbf{S d}\right)$ can be identified with a subset of $\bar{\Omega}_{E(G)} \mathbf{S}$ (proposition 2.3). Lemma 5.7 in [4] states an opposite relation, in the sense that, for any finite set $X$ and for each $\pi \in \bar{\Omega}_{X} \mathbf{S}$ we can associate an edge implicit operation in $\bar{\Omega}_{\mathcal{A}_{\pi}} \mathbf{S d}$, again denoted by $\pi$. Note that, if $\left(w_{n}\right)_{n}$ is a sequence of words in $X^{+}$converging to $\pi$, then there is an integer $p$ such that, for all $n \geq p, w_{n}$ labels a path in $\mathcal{A}_{\pi}$ from the initial vertex to the final one and all edges of $\mathcal{A}_{\pi}$ occur in $w_{n}$. For example, let $\pi=x y^{\omega} z x$ and consider the sequence $\left(x y^{n !} z x\right)_{n}$ that converges to $\pi$. The graph $\mathcal{A}_{\pi}$ is 


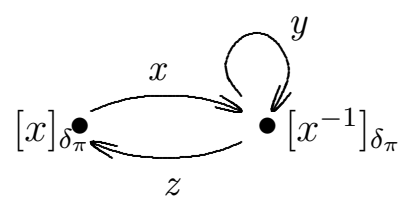

and, for every $n, x y^{n !} z x$ denotes a path from $[x]_{\delta_{\pi}}$ to $\left[x^{-1}\right]_{\delta_{\pi}}$. The limit of the sequence of paths $\left(x y^{n !} z x\right)_{n}$ is the edge implicit operation denoted by $x y^{\omega} z x$.

Theorem 2.3. [4, theorem 5.9] Let $\mathbf{V}$ be a pseudovariety of semigroups such that $B_{2} \in \mathbf{V}$. If $\mathbf{V}=\llbracket u_{i}=v_{i}: i \in I \rrbracket$ then $g \mathbf{V}=\llbracket\left(u_{i}=v_{i}, \mathcal{A}_{u_{i}}\right): i \in I \rrbracket$.

Corollary 2.4. The pseudovariety $g \mathbf{V}\left(B_{2}\right)$ is the pseudovariety $\llbracket\left(x^{3}=x^{2}, \mathcal{A}_{x^{2}}\right),\left(x^{2} y^{2}=y^{2} x^{2}, \mathcal{A}_{x^{2} y^{2}}\right),\left(x(y x)^{2}=x y x, \mathcal{A}_{x y x}\right) \rrbracket$.

\section{THE SEMIDIRECT POWERS OF V $\left(B_{2}\right)$}

The semidirect powers of a pseudovariety of semigroups $\mathbf{W}$ are:

- $\mathbf{W}^{0}=\llbracket x=y \rrbracket$ the pseudovariety of trivial semigroups;

- $\mathbf{W}^{n}=\mathbf{W} * \mathbf{W}^{n-1}\left(=\mathbf{W}^{n-1} * \mathbf{W}\right)$, for every $n \geq 1$.

So, $\mathbf{W}^{1}=\mathbf{W}, \mathbf{W}^{n-1} \subseteq \mathbf{W}^{n}$, the family $\left(\mathbf{W}^{n}\right)_{n \geq 0}$ is a chain and $\bigcup_{n>0} \mathbf{W}^{n}$ is the s.c. pseudovariety generated by $\mathbf{W}$, usually represented by $\mathbf{W}^{*}$. The computation of the semidirect powers of $\mathbf{V}\left(B_{2}\right)$ can be done recursively using in each step theorems 2.1 and 2.3 .

Proposition 3.1. Let $G$ be a graph with a vertex $v$ such that, for every $v^{\prime} \in V(G) \backslash\{v\}, G^{+}\left(v, v^{\prime}\right) \neq \emptyset$. A labeling

$$
\left(\left(\pi_{q}\right)_{q \in V(G)}, \varepsilon^{\prime}: E(G) \longrightarrow \bar{\Omega}_{X} \mathbf{S}\right)
$$

is compatible with a pseudovariety $\mathbf{V}$ if and only if, for every $q \in V(G)$ and $w \in G^{+}(v, q), \mathbf{V} \models \pi_{v} \varepsilon(w)=\pi_{q}$, where $\varepsilon: \bar{\Omega}_{G} \mathbf{S d} \longrightarrow \bar{\Omega}_{X} \mathbf{S}$ is the continuous morphism of semigroupoids constant on vertices and defined on edges by: $\varepsilon(s)=\varepsilon^{\prime}(s)$, for every $s \in E(G)$.

\section{Proof}

The condition is obviously necessary. In order to prove the inverse implication, let $s \in E(G)$ and $w_{\alpha(s)} \in G^{+}(v, \alpha(s))$. By hypothesis

$$
\mathbf{V} \models \pi_{v} \varepsilon\left(w_{\alpha(s)}\right)=\pi_{\alpha(s)} \text { and } \mathbf{V} \models \pi_{v} \varepsilon\left(w_{\alpha(s)} s\right)=\pi_{\omega(s)} .
$$

Hence, $\mathbf{V} \models \pi_{\alpha(s)} \varepsilon(s)=\pi_{\omega(s)}$. 
Corollary 3.2. Let $G$ be a graph in the conditions of proposition 3.1, $\pi \in \bar{\Omega}_{X} \mathbf{S}$ and $\varepsilon^{\prime}: E(G) \longrightarrow \bar{\Omega}_{X} \mathbf{S}$ such that, for every $q \in V(G)$ and $w_{1}, w_{2} \in G^{+}(v, q), \mathbf{V} \models \pi \varepsilon\left(w_{1}\right)=\pi \varepsilon\left(w_{2}\right)$ and, in case $v=q$, $\mathbf{V} \models \pi \varepsilon\left(w_{1}\right)=\pi$, where $\varepsilon: \bar{\Omega}_{G} \mathbf{S d} \longrightarrow \bar{\Omega}_{X} \mathbf{S}$ is the continuous morphism of semigroupoids constant on vertices and defined on edges by: $\varepsilon(s)=\varepsilon^{\prime}(s)$, for every $s \in E(G)$. Then, the set of the labelings of the form $\left(\left(\pi_{q}\right)_{q \in V(G)}, \varepsilon^{\prime}\right)$ such that $\pi_{v}=\pi$ which are compatible with $\mathbf{V}$ is nonempty.

\section{Proof}

Let $\varphi: \bar{\Omega}_{X} \mathbf{S} \longrightarrow \bar{\Omega}_{X} \mathbf{V}$ be the projection morphism. If we choose $\pi_{v}=\pi$ and, for each $q \in V(G) \backslash\{v\}$ and some $w_{1} \in G^{+}(v, q)$, $\pi_{q} \in \varphi^{-1}\left(\varphi\left(\pi \varepsilon\left(w_{1}\right)\right)\right)$, then we obtain a family $\left(\pi_{q}\right)_{q \in V(G)}$ such that $\left(\left(\pi_{q}\right)_{q \in V(G)}, \varepsilon^{\prime}\right)$ define a labeling of $G$. By proposition 3.1, such labeling is compatible with $\mathbf{V}$. Making all possible choices we obtain the set of labelings referred to in the statement.

In the conditions of the corollary 3.2 we will say that $\pi$ and $\varepsilon$ define a set of labelings of $G$ by $\bar{\Omega}_{X} \mathbf{S}$ compatible with $\mathbf{V}$ or, more precisely, $\pi$ and $\varepsilon$ define the set of labelings referred to in the proof.

This result applied to the calculation of the pseudovariety $\mathbf{V}^{2}\left(B_{2}\right)$, using theorem 2.1 and the pseudoidentity basis $\Gamma$ of $g \mathbf{V}\left(B_{2}\right)$ calculated in corollary 2.4 , leads to the conclusion that

$$
\begin{aligned}
\Sigma=\{ & \pi \varepsilon(u)=\pi \varepsilon(v): X \text { is a finite set, } \pi \in\left(\bar{\Omega}_{X} \mathbf{S}\right)^{1} \\
& \text { and } \varepsilon: \bar{\Omega}_{\mathcal{A}_{u}} \mathbf{S d} \longrightarrow \bar{\Omega}_{X} \mathbf{S} \text { define a set of labelings } \\
& \text { compatible with } \left.\mathbf{V}\left(B_{2}\right) \text {, and }\left(u=v, \mathcal{A}_{u}\right) \in \Gamma\right\}
\end{aligned}
$$

is a pseudoidentity basis of $\mathbf{V}^{2}\left(B_{2}\right)$.

Note that for $\left(u=v, \mathcal{A}_{u}\right) \in \Sigma$ and all labelings member of the set of labelings defined by fixed $\pi$ and $\varepsilon$ corresponds only one pseudoidentity on the basis of $\mathbf{V}^{2}\left(B_{2}\right)$. The verification that a labeling is compatible with $\mathbf{V}\left(B_{2}\right)$ is based on theorem 2.2 .

In order to give a pseudoidentity basis of pseudovarieties of the form $\mathbf{V}^{n}\left(B_{2}\right)$ we need to prove the following lemmas.

Lemma 3.3. Let $\pi \in \bar{\Omega}_{X} \mathbf{S}$ and $\rho$ be a factor of $\pi$. Then there is a graph morphism $\varphi_{\rho, \pi}: \mathcal{A}_{\rho} \longrightarrow \mathcal{A}_{\pi}$ such that $\varphi_{\rho, \pi}\left(\mathcal{A}_{\rho}\right)$ is the support graph of $C(\rho)$.

\section{Proof}


Recall that $C(\rho) \subseteq C(\pi)=E\left(\mathcal{A}_{\pi}\right)$. Consider the graph morphism

$$
\begin{aligned}
\varphi_{\rho, \pi}: \mathcal{A}_{\rho} & \longrightarrow \mathcal{A}_{\pi} \\
{[x]_{\delta_{\rho}} } & \longmapsto[x]_{\delta_{\pi}}
\end{aligned}
$$

Since $\delta_{\rho} \subseteq \delta_{\pi}, \varphi_{\rho, \pi}$ is a graph morphism injective on edges.

Lemma 3.4. Let $\pi, u \in \bar{\Omega}_{X} \mathbf{S}$ and $\varepsilon: \bar{\Omega}_{\mathcal{A}_{u}} \mathbf{S d} \longrightarrow \bar{\Omega}_{X} \mathbf{S}$ be such that $\pi$ and $\varepsilon$ define a set of labelings of $\mathcal{A}_{u}$ compatible with $\mathbf{V}\left(B_{2}\right)$. If, for some $\pi_{a} \in \bar{\Omega}_{X} \mathbf{S}, \pi_{b} \in\left(\bar{\Omega}_{X} \mathbf{S}\right)^{1}$,

$$
\pi=\pi_{a} \pi_{b} \text { and } C\left(\pi_{a}\right) \cap C\left(\pi_{b} \varepsilon(u)\right)=\emptyset,
$$

then $\pi_{b}$ and $\varepsilon$ define a set of labelings of $\mathcal{A}_{u}$ compatible with $\mathbf{V}\left(B_{2}\right)$.

\section{Proof}

Let $v$ be the initial vertex of $\mathcal{A}_{u}, q \in V\left(\mathcal{A}_{u}\right)$ and $w, w^{\prime} \in \mathcal{A}_{u}(v, q)$. Since $\pi=\pi_{a} \pi_{b}$, the graph $\mathcal{A}_{\pi \varepsilon(w)}$ is the union of the subgraphs $\varphi_{\pi_{a}, \pi \varepsilon(w)}\left(\mathcal{A}_{\pi_{a}}\right)$ and $\varphi_{\pi_{b} \varepsilon(w), \pi \varepsilon(w)}\left(\mathcal{A}_{\pi_{b} \varepsilon(w)}\right)$. These two subgraphs have only one common vertex: the image under $\varphi_{\pi_{a}, \pi \varepsilon(w)}$ of the final vertex of $\mathcal{A}_{\pi_{a}}$ is equal to the image under $\varphi_{\pi_{b} \varepsilon(w), \pi \varepsilon(w)}$ of the initial vertex of $\mathcal{A}_{\pi_{b} \varepsilon(w)}$. Otherwise, the graph $\mathcal{A}_{\pi \varepsilon\left(w^{\prime}\right)}$ is the union of the subgraphs $\varphi_{\pi_{a}, \pi \varepsilon\left(w^{\prime}\right)}\left(\mathcal{A}_{\pi_{a}}\right)$ and $\varphi_{\pi_{b} \varepsilon\left(w^{\prime}\right), \pi \varepsilon\left(w^{\prime}\right)}\left(\mathcal{A}_{\pi_{b} \varepsilon\left(w^{\prime}\right)}\right)$, ant these two subgraphs have only one common vertex: the image under $\varphi_{\pi_{a}, \pi \varepsilon\left(w^{\prime}\right)}$ of the final vertex of $\mathcal{A}_{\pi_{a}}$ which is the image under $\varphi_{\pi_{b} \varepsilon(w), \pi \varepsilon(w)}$ of the initial vertex of $\mathcal{A}_{\pi_{b} \varepsilon(w)}$. Moreover, by condition (1), the graph morphisms $\varphi_{\pi_{a}, \pi \varepsilon(w)}, \varphi_{\pi_{a}, \pi \varepsilon\left(w^{\prime}\right)}, \varphi_{\pi_{b} \varepsilon(w), \pi \varepsilon(w)}$ and $\varphi_{\pi_{b} \varepsilon\left(w^{\prime}\right), \pi \varepsilon\left(w^{\prime}\right)}$ are injective on edges and on vertices.

By hypothesis $\pi$ and $\varepsilon$ define a set of labelings of $\mathcal{A}_{u}$ compatible with $\mathbf{V}\left(B_{2}\right)$, so $\mathcal{A}_{\pi \varepsilon(w)}=\mathcal{A}_{\pi \varepsilon\left(w^{\prime}\right)}$ and the final vertices are the same which means that $[t(\pi \varepsilon(w))]_{\delta_{\pi \varepsilon(w)}^{-1}}^{-1}=\left[t\left(\pi \varepsilon\left(w^{\prime}\right)\right)\right]_{\delta_{\pi \varepsilon\left(w^{\prime}\right)}}^{-1}$. Consequently, $\varphi_{\pi_{b} \varepsilon(w), \pi \varepsilon(w)}\left(\mathcal{A}_{\pi_{b} \varepsilon(w)}\right)=\varphi_{\pi_{b} \varepsilon\left(w^{\prime}\right), \pi \varepsilon\left(w^{\prime}\right)}\left(\mathcal{A}_{\pi_{b} \varepsilon\left(w^{\prime}\right)}\right)$, which implies $\mathcal{A}_{\pi_{b} \varepsilon(w)}=$ $\mathcal{A}_{\pi_{b} \varepsilon\left(w^{\prime}\right)}$, and the initial and the final vertices of $\mathcal{A}_{\pi_{b} \varepsilon(w)}$ and $\mathcal{A}_{\pi_{b} \varepsilon\left(w^{\prime}\right)}$ are the same, respectively, because

$$
\begin{aligned}
{\left[i\left(\pi_{b} \varepsilon(w)\right)\right]_{\delta_{\pi_{b} \varepsilon(w)}} } & =\varphi_{\pi_{b} \varepsilon(w), \pi \varepsilon(w)}^{-1}\left(\left[t\left(\pi_{a}\right)^{-1}\right]_{\delta_{\pi \varepsilon(w)}}\right)= \\
& =\varphi_{\pi_{b} \varepsilon\left(w^{\prime}\right), \pi \varepsilon\left(w^{\prime}\right)}^{-1}\left(\left[t\left(\pi_{a}\right)^{-1}\right]_{\delta_{\pi \varepsilon\left(w^{\prime}\right)}}\right)=\left[i\left(\pi_{b} \varepsilon\left(w^{\prime}\right)\right)\right]_{\delta_{\pi_{b} \varepsilon\left(w^{\prime}\right)}}
\end{aligned}
$$

and

$$
\begin{aligned}
{\left[t\left(\pi_{b} \varepsilon(w)\right)\right]_{\delta_{\pi_{b} \varepsilon(w)}}^{-1} } & =\varphi_{\pi_{b} \varepsilon(w), \pi \varepsilon(w)}^{-1}\left([t(\pi \varepsilon(w))]_{\delta_{\pi \varepsilon(w)}}^{-1}\right)= \\
& =\varphi_{\pi_{b} \varepsilon\left(w^{\prime}\right), \pi \varepsilon\left(w^{\prime}\right)}^{-1}\left(\left[t\left(\pi \varepsilon\left(w^{\prime}\right)\right)\right]_{\delta_{\pi \varepsilon\left(w^{\prime}\right)}}^{-1}\right)=\left[t\left(\pi_{b} \varepsilon\left(w^{\prime}\right)\right)\right]_{\delta_{\pi_{b} \varepsilon\left(w^{\prime}\right)}^{-1}}^{-1}
\end{aligned}
$$


For $\pi, \pi_{a}, u \in \bar{\Omega}_{X} \mathbf{S}, \pi_{b} \in \bar{\Omega}_{X} \mathbf{S}^{1}$ and $\varepsilon: \bar{\Omega}_{\mathcal{A}_{u}} \mathbf{S d} \longrightarrow \bar{\Omega}_{X} \mathbf{S}$ such that $\pi=\pi_{a} \pi_{b}$, the pseudoidentity $\pi \varepsilon(u)=\pi \varepsilon(v)$ is a consequence of $\pi_{b} \varepsilon(u)=\pi_{b} \varepsilon(v)$. Hence, for example, as a pseudoidentity basis of $\mathbf{V}^{2}\left(B_{2}\right)$ we can consider the set $\Sigma_{2}$ of the pseudoidentities such as those in $\Sigma$ and such that the values of $\pi$ do not satisfy the condition (1).

Lemma 3.5. Let $\pi \in \bar{\Omega}_{X} \mathbf{S}$ such that $i(\pi)=t(\pi)$ and $\pi \notin X$. Then $\mathcal{A}_{\pi}$ is strongly connected.

\section{Proof}

Note that, for every $u \in \bar{\Omega}_{X} \mathbf{S}$ and for each $x \in C(u)$, there is exactly one edge of $\mathcal{A}_{u}$ defined by $x$, which belongs to a path defined by a word factor of $u$.

Let $\left(w_{n}\right)_{n}$ be a sequence of words converging to $\pi$. Then there is $p \in \mathbb{N}$ such that, for all $n \geq p, i(\pi)=i\left(w_{n}\right)=t\left(w_{n}\right), B_{2} \models w_{n}=\pi$ and, consequently, $\left(w_{n}\right)_{n \geq p}$ is a sequence of paths of $\mathcal{A}_{\pi}$. Moreover, $\mathcal{A}_{\pi}$ is the support of each $w_{n}$ (no proper subgraph of $\mathcal{A}_{\pi}$ contains all edges of $\left.w_{n}\right)$ and $i(\pi)$ is the initial and final edge of $w_{n}$. For $n \geq p$, there is $w_{n}^{\prime} \in E\left(\mathcal{A}_{\pi}^{+}\right)$such that $w_{n}=w_{n}^{\prime} i(\pi)$ and, consequently, the support graph of $w_{n}^{\prime}$ is $\mathcal{A}_{\pi}$ and $\alpha\left(w_{n}^{\prime}\right)=\omega\left(w_{n}^{\prime}\right)=\alpha\left(w_{n}\right)$ is the initial vertex of $\mathcal{A}_{\pi}$. Hence $w_{n}^{\prime}$ is a path of $\mathcal{A}_{\pi}$ such that, for each $v \in V\left(\mathcal{A}_{\pi}\right)$, there are factors $u_{v} \in \mathcal{A}_{\pi}^{+}\left(\alpha\left(w_{n}^{\prime}\right), v\right)$ and $u^{v} \in \mathcal{A}_{\pi}^{+}\left(v, \alpha\left(w_{n}^{\prime}\right)\right)$. So, for every $v, v^{\prime} \in V\left(\mathcal{A}_{\pi}\right), u^{v} u_{v^{\prime}} \in \mathcal{A}_{\pi}^{+}\left(v, v^{\prime}\right)$ and $u^{v^{\prime}} u_{v} \in \mathcal{A}_{\pi}^{+}\left(v^{\prime}, v\right)$.

Note that, if $G_{1}$ and $G_{2}$ are strongly connected subgraphs of a graph $G$, such that $V(G)=V\left(G_{1}\right) \cup V\left(G_{2}\right)$ and $V\left(G_{1}\right) \cap V\left(G_{2}\right) \neq \emptyset$, then $G$ is strongly connected.

Lemma 3.6. Let $X$ be a finite set and $\pi \in \bar{\Omega}_{X} \mathbf{S}$ such that $\pi=\pi_{1} \pi_{2} \pi_{3}$, where $\pi_{1}, \pi_{2}, \pi_{3} \in\left(\bar{\Omega}_{X} \mathbf{S}\right)^{1}$, and suppose $\mathcal{A}_{\pi_{1} \pi_{2}}$ and $\mathcal{A}_{\pi_{2} \pi_{3}}$ are strongly connected graphs. Then, $\mathcal{A}_{\pi}$ is strongly connected.

\section{Proof}

The graph $\mathcal{A}_{\pi}$ is the union of $\varphi_{\pi_{1} \pi_{2}, \pi}\left(\mathcal{A}_{\pi_{1} \pi_{2}}\right)$ and $\varphi_{\pi_{2} \pi_{3}, \pi}\left(\mathcal{A}_{\pi_{2} \pi_{3}}\right)$, which are strongly connected graphs and both contain the vertex $\left[i\left(\pi_{2}\right)\right]_{\delta_{\pi}}$, if $\pi_{2} \neq 1$, or the vertex $\left[t\left(\pi_{1}\right)^{-1}\right]_{\delta_{\pi}}=\left[i\left(\pi_{3}\right)\right]_{\delta_{\pi}}$, if $\pi_{2}=1$.

Lemma 3.7. Let $u \in \bar{\Omega}_{X} \mathbf{S}$. If $\pi \in \bar{\Omega}_{X} \mathbf{S}$ and $\varepsilon: \bar{\Omega}_{\mathcal{A}_{u}} \mathbf{S d} \longrightarrow \bar{\Omega}_{X} \mathbf{S}$ is a continuous morphism of semigroupoids, which define a set of labelings of $\mathcal{A}_{u}$ compatible with $\mathbf{V}\left(B_{2}\right)$, then

i. if $\mathcal{A}_{u}$ is strongly connected then $\mathcal{A}_{\pi \varepsilon(u)}=\mathcal{A}_{\pi}$; 
ii. if $\pi$ does not have a decomposition as in (1) and $C(\varepsilon(u)) \subseteq C(\pi)$, then $\mathcal{A}_{\pi \varepsilon(u)}$ is strongly connected.

\section{Proof}

If $\mathcal{A}_{u}$ is strongly connected then there is $u^{\prime} \in \mathcal{A}_{u}^{*}\left(\left[t(u)^{-1}\right]_{\delta_{u}},[i(u)]_{\delta_{u}},\right)$. For any such word $u^{\prime}, B_{2} \models \pi \varepsilon(u) \varepsilon\left(u^{\prime}\right)=\pi$ and, consequently, $C(\varepsilon(u)) \subseteq C(\pi)$ and $\delta_{\pi} \subseteq \delta_{\pi \varepsilon(u)} \subseteq \delta_{\pi \varepsilon(u) \varepsilon\left(u^{\prime}\right)}=\delta_{\pi}$. Hence, $\mathcal{A}_{\pi \varepsilon(u)}=\mathcal{A}_{\pi}$.

Now, suppose $\pi$ does not have a decomposition as in (1) and $C(\varepsilon(u)) \subseteq C(\pi)$. If $i(\pi)=t(\varepsilon(u))$ then lemma 3.5 leads to the conclusion that $\mathcal{A}_{\pi \varepsilon(u)}$ is strongly connected. If $i(\pi) \neq t(\varepsilon(u))$ then, since $C(\varepsilon(u)) \subseteq C(\pi)$, there are $\pi_{1}, \pi_{0}, u_{0} \in\left(\bar{\Omega}_{X} \mathbf{S}\right)^{1}$ such that

$$
\pi \varepsilon(u)=l_{1} \pi_{1} z \pi_{0} u_{0} z
$$

where $i(\pi)=l_{1}, t(\varepsilon(u))=z$ and $u_{0} z=\varepsilon(u), z \notin C\left(l_{1} \pi_{1}\right)$ and $C\left(l_{1} \pi_{1}\right) \cap$ $C\left(\pi_{0} u_{0}\right) \neq \emptyset$ since $\pi$ can not be factorized as in (1).

If $l_{1} \in C\left(\pi_{0} u_{0}\right)$ then $\pi \varepsilon(u)$ is of the form $l_{1} \cdots z \cdots l_{1} \cdots z$ and, by lemmas 3.5 and 3.6, $\mathcal{A}_{\pi \varepsilon(u)}$ is strongly connected. Otherwise, there exist $\pi_{1}^{\prime}, \pi_{2} \in\left(\bar{\Omega}_{X} \mathbf{S}\right)^{1}$ and $l_{2} \in X$ such that $\pi_{1}=\pi_{1}^{\prime} l_{1} \pi_{2}, l_{1} \notin C\left(\pi_{2}\right)$, $l_{2} \in C\left(\pi_{1}^{\prime}\right) \cap C\left(\pi_{2} z \pi_{0} u_{0} z\right)$, and

$$
\pi \varepsilon(u)=l_{1} \pi_{1}^{\prime} l_{1} \pi_{2} z \pi_{0} u_{0} z .
$$

If $l_{2} \in C\left(\pi_{0} u_{0}\right)$ then $\pi \varepsilon(u)$ is of the form $l_{1} \cdots l_{2} \cdots l_{1} \cdots z \cdots l_{2} \cdots z$ and, using lemmas 3.5 and 3.6, we conclude that $\mathcal{A}_{\pi \varepsilon(u)}$ is strongly connected. If $l_{2} \notin C\left(\pi_{0} u_{0}\right)$ then there are $\pi_{2}^{\prime}, \pi_{3} \in\left(\bar{\Omega}_{X} \mathbf{S}\right)^{1}$ such that $\pi_{2}=\pi_{2}^{\prime} l_{2} \pi_{3}, l_{2} \notin C\left(\pi_{3}\right) \subset C\left(\pi_{2}\right)$, and

$$
\pi \varepsilon(u)=l_{1} \pi_{1}^{\prime} l_{1} \pi_{2}^{\prime} l_{2} \pi_{3} z \pi_{0} u_{0} z .
$$

Hence, $\mathcal{A}_{l_{1} \cdots l_{2} \cdots l_{1} \pi_{2}^{\prime} l_{2}}$ is strongly connected and there is $l_{3} \in X$ such that $l_{3} \in C\left(\pi_{1}^{\prime} l_{1} \pi_{2}^{\prime}\right) \cap C\left(\pi_{3} z \pi_{0} u_{0} z\right)$.

Since $C(\pi)$ is finite, after a finite number of steps $i$, either we conclude that $\mathcal{A}_{\pi \varepsilon(u)}$ is strongly connected or $\pi_{i+1}=1$. In the second case, $\mathcal{A}_{l_{1} \cdots l_{i-1} \pi_{i}^{\prime} l_{i}}$ is strongly connected,

$$
\pi \varepsilon(u)=l_{1} \pi_{1}^{\prime} l_{1} \pi_{2}^{\prime} l_{2} \cdots l_{i-1} \pi_{i}^{\prime} l_{i} z \pi_{0} u_{0} z
$$

and, for every $j \in\{1, \ldots, i\}, l_{j} \notin C\left(z \pi_{0} u_{0} z\right)$. Since $\pi$ can not be factorized as in (1), then, there is $y \in C\left(l_{1} \pi_{1}^{\prime} l_{1} \pi_{2}^{\prime} l_{2} \cdots l_{i-1} \pi_{i}^{\prime}\right) \cap C\left(z \pi_{0} u_{0} z\right)$, and $\mathcal{A}_{\pi \varepsilon(u)}$ is the union of $\varphi_{l_{1} \cdots \pi_{i}^{\prime} l_{i}, \pi \varepsilon(u)}\left(\mathcal{A}_{l_{1} \cdots \pi_{i}^{\prime} l_{i}}\right)$ and $\varphi_{z \pi_{0} u_{0} z, \pi \varepsilon(u)}\left(\mathcal{A}_{z \pi_{0} u_{0} z}\right)$. By lemmas 3.5 and 3.6, we conclude that $\mathcal{A}_{\pi \varepsilon(u)}$ is strongly connected. 
Applying theorem 2.3 and the above lemmas to the calculation of $g \mathbf{V}^{2}\left(B_{2}\right)$, we conclude that

$$
g \mathbf{V}^{2}\left(B_{2}\right)=\llbracket\left(\pi \varepsilon(u)=\pi \varepsilon(v), \mathcal{A}_{\pi}\right): \pi \varepsilon(u)=\pi \varepsilon(v) \in \Sigma_{2} \rrbracket
$$

where, in each case, $\mathcal{A}_{\pi \varepsilon(u)}=\mathcal{A}_{\pi}$ is a strongly connected graph.

Theorem 3.8. For each $n \geq 1$, let $\Sigma_{n}$ be the set of all pseudoidentities of the form

$\pi_{n-1} \varepsilon_{n-1}\left(\pi_{n-2} \cdots \varepsilon_{2}\left(\pi_{1} \varepsilon_{1}(u)\right) \cdots\right)=\pi_{n-1} \varepsilon_{n-1}\left(\pi_{n-2} \cdots \varepsilon_{2}\left(\pi_{1} \varepsilon_{1}(v)\right) \cdots\right)$,

where

- $(u=v) \in\left\{x^{3}=x^{2}, x^{2} y^{2}=y^{2} x^{2}, x(y x)^{2}=x y x\right\}$,

- for $i=1, \ldots, n-1, X_{i}$ is a finite set, $\pi_{i} \in \bar{\Omega}_{X_{i}} \mathbf{S}$ and

$\varepsilon_{i}: \bar{\Omega}_{\mathcal{A}_{\pi_{i-1}}} \mathbf{S d} \longrightarrow \bar{\Omega}_{X_{i}} \mathbf{S}$ define a set of labelings of $\mathcal{A}_{\pi_{i-1}}$ compatible with $\mathbf{V}\left(B_{2}\right)$, such that each $\pi_{i}$ can not be factorized as in (1) relatively to $\varepsilon_{i}\left(\pi_{i-1} \cdots \varepsilon_{1}(u) \cdots\right)$ (by $\pi_{0}$ we mean $u$ ).

Then $\Sigma_{n}$ is a pseudoidentity basis of $\mathbf{V}^{n}\left(B_{2}\right)$, and

$$
\mathcal{A}_{\pi_{n-1}}=\mathcal{A}_{\pi_{n-1} \varepsilon_{n-1}\left(\pi_{n-2} \cdots \varepsilon_{2}\left(\pi_{1} \varepsilon_{1}(u)\right) \ldots\right)}
$$

is strongly connected.

\section{Proof}

The proof is by induction on $n$. The statement is valid for $n=1,2$, as we have noted before.

By induction hypothesis suppose that, for some $p \geq 1$, the statement is valid. So, if $\Gamma_{p}$ is the set

$$
\begin{gathered}
\left\{\left(\pi_{p-1} \varepsilon_{p-1}\left(\pi_{p-2} \cdots \varepsilon_{1}(u) \ldots\right)=\pi_{p-1} \varepsilon_{p-1}\left(\pi_{p-2} \cdots \varepsilon_{1}(v) \ldots\right), \mathcal{A}_{\pi_{p-1}}\right):\right. \\
\left.\pi_{p-1} \varepsilon_{p-1}\left(\pi_{p-2} \cdots \varepsilon_{1}(u) \ldots\right)=\pi_{p-1} \varepsilon_{p-1}\left(\pi_{p-2} \cdots \varepsilon_{1}(v) \ldots\right) \in \Sigma_{p}\right\},
\end{gathered}
$$

then $g \mathbf{V}^{p}\left(B_{2}\right)=\llbracket \Gamma_{p} \rrbracket$ and, by lemma 3.7, the graph $\mathcal{A}_{\pi_{p-1}}$ is equal to the strongly connected graph $\mathcal{A}_{\pi_{p-1} \varepsilon_{p-1}\left(\pi_{p-2} \cdots \varepsilon_{1}(u) \ldots\right)}$. Hence, by theorem 2.1, we conclude that the pseudovariety $\mathbf{V}^{p+1}\left(B_{2}\right)$ is defined by the pseudoidentities of the form

$$
\pi_{p} \varepsilon_{p}\left(u_{p}\right)=\pi_{p} \varepsilon_{p}\left(v_{p}\right)
$$

where

- $\left(u_{p}=v_{p}, \mathcal{A}_{\pi_{p-1}}\right) \in \Gamma_{p}$,

- $X_{p}$ a finite set, $\pi_{p} \in \bar{\Omega}_{X_{p}} \mathbf{S}$ and $\varepsilon_{p}: \bar{\Omega}_{\mathcal{A}_{\pi_{p-1}}} \mathbf{S d} \longrightarrow \bar{\Omega}_{X_{p}} \mathbf{S}$ define a set of labelings of $\mathcal{A}_{\pi_{p-1}}$ compatible with $\mathbf{V}\left(B_{2}\right)$ and, by lemma 3.4, $\pi_{p}$ can not be factorized as in (1) relatively to $\varepsilon_{p}\left(u_{p}\right)$. 
The graph $\mathcal{A}_{\pi_{p-1}}\left(=\mathcal{A}_{u_{p}}\right)$ is strongly connected, by induction hypothesis, and then, by lemma $3.7, \mathcal{A}_{\pi_{p}}$ is equal to $\mathcal{A}_{\pi_{p} \varepsilon_{p}\left(u_{p}\right)}$ which is strongly connected.

Corollary 3.9. The pseudovariety of semigroupoids $g \mathbf{V}^{n}\left(B_{2}\right)$ is defined by pseudoidentities over strongly connected graphs, for $n \geq 1$.

Using the same arguments, we can prove that some pseudoidentities hold in $\mathbf{V}^{*}\left(B_{2}\right)$. As an example, consider an alphabet $X$ such that $\{x, y, z, t\} \subseteq X$ and the pseudoidentity

$$
(z x y x t z)^{\omega} x(y x)^{2}=(z x y x t z)^{\omega} x y x
$$

which holds in $\mathbf{V}\left(B_{2}\right)$, by theorem 2.2 . The graph $\mathcal{A}_{(z x y x t z)^{\omega}{ }_{x y x}}$ is presented in the next picture, where $v_{1}=\left\{z^{-1}, z, x, t^{-1}, y^{-1}\right\}$ is the initial vertex and $v_{2}=\left\{x^{-1}, t, y\right\}$ is the final vertex.

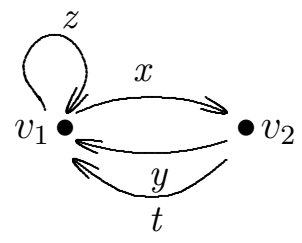

Suppose that the pseudoidentity holds in $\mathbf{V}^{i}\left(B_{2}\right)$, for some $i \geq 1$. The labeling defined by $\pi=(z x y x t z)^{\omega}$ and $\varepsilon: \bar{\Omega}_{\mathcal{A}_{(z x y x t z)}{ }_{x y x}} \mathbf{S d} \longrightarrow \bar{\Omega}_{X} \mathbf{S}$ such that $\varepsilon(l)=l$, for every $l \in\{x, y, z, t\}$, is compatible with $\mathbf{V}\left(B_{2}\right)$, since $B_{2} \models \pi z=\pi x y=\pi x t=\pi$. Hence, by theorem 2.1,

$$
\mathbf{V}^{i+1}\left(B_{2}\right) \models(z x y x t z)^{\omega} x(y x)^{2}=(z x y x t z)^{\omega} x y x .
$$

So, for every natural $n, \mathbf{V}^{n}\left(B_{2}\right) \models(z x y x t z)^{\omega} x(y x)^{2}=(z x y x t z)^{\omega} x y x$ and, consequently,

$$
\mathbf{V}^{*}\left(B_{2}\right) \models(z x y x t z)^{\omega} x(y x)^{2}=(z x y x t z)^{\omega} x y x .
$$

\section{DECIDABILITY OF $\mathbf{V}^{*}\left(B_{2}\right)$}

A pseudovariety $\mathbf{V}$ is decidable if there is an algorithm to test whether a finite semigroup is or is not in $\mathbf{V}$. Theorem 3.8 does not lead to an effective construction of a basis of pseudoidentities for the semidirect powers of $\mathbf{V}\left(B_{2}\right)$, but it nevertheless suggests a proof of decidability of $\mathbf{V}^{*}\left(B_{2}\right)$.

Theorem 4.1. Let $S$ be a finite semigroup with $m$ elements. Then, $S \in \mathbf{V}^{*}\left(B_{2}\right)$ if and only if $S \in \mathbf{V}^{m+2}\left(B_{2}\right)$. 


\section{Proof}

To prove the non trivial implication, let $S \in \mathbf{V}^{*}\left(B_{2}\right)$. Then there is $k \geq 1$ such that $S \in \mathbf{V}^{k}\left(B_{2}\right)$. If $k \leq m+2$, then $\mathbf{V}^{k}\left(B_{2}\right) \subseteq \mathbf{V}^{m+2}\left(B_{2}\right)$ and, consequently, $S \in \mathbf{V}^{m+2}\left(B_{2}\right)$.

Suppose $k>m+2$. Proving that $S \in \mathbf{V}^{m+2}\left(B_{2}\right)$ is equivalent to proving that the pseudoidentities of a basis of $\mathbf{V}^{m+2}\left(B_{2}\right)$ hold in $S$. By theorem 3.8, we may take all pseudoidentities of the form:

$$
\pi_{m+1} \varepsilon_{m+1}\left(\pi_{m} \cdots \varepsilon_{2}\left(\pi_{1} \varepsilon_{1}(u)\right) \ldots\right)=\pi_{m+1} \varepsilon_{m+1}\left(\pi_{m} \cdots \varepsilon_{2}\left(\pi_{1} \varepsilon_{1}(v)\right) \ldots\right)
$$

where $u, v, \pi_{i}, \varepsilon_{i}$ are in the conditions described in the theorem 3.8, for $i=1,2, \ldots, m+1$. Let $\lambda$ denote one of these pseudoidentities and, for $m+1 \geq i \geq j \geq 1$,

$$
\begin{aligned}
& p_{i, j}=\varepsilon_{m+1} \circ \cdots \circ \varepsilon_{i+1}\left(\pi_{i} \varepsilon_{i}\left(\pi_{i-1} \cdots \varepsilon_{j+1}\left(\pi_{j}\right) \ldots\right)\right) \\
& p_{i, 0}^{u}=\varepsilon_{m+1} \circ \cdots \circ \varepsilon_{i+1}\left(\pi_{i} \varepsilon_{i}\left(\pi_{i-1} \cdots \varepsilon_{1}(u) \ldots\right)\right) \\
& p_{i, 0}^{v}=\varepsilon_{m+1} \circ \cdots \circ \varepsilon_{i+1}\left(\pi_{i} \varepsilon_{i}\left(\pi_{i-1} \cdots \varepsilon_{1}(v) \ldots\right)\right) \\
& q_{i, j}=\pi_{i} \varepsilon_{i}\left(\pi_{i-1} \cdots \varepsilon_{j+1}\left(\pi_{j}\right) \ldots\right) \\
& q_{i, 0}^{u}=\pi_{i} \varepsilon_{i}\left(\pi_{i-1} \cdots \varepsilon_{1}(u) \ldots\right) \\
& q_{i, 0}^{v}=\pi_{i} \varepsilon_{i}\left(\pi_{i-1} \cdots \varepsilon_{1}(v) \ldots\right)
\end{aligned}
$$

Note that $\lambda$ is the pseudoidentity $q_{m+1,0}^{u}=q_{m+1,0}^{v}$.

The expressions

$$
\begin{aligned}
& p_{1,1}=\varepsilon_{m+1} \circ \cdots \circ \varepsilon_{2}\left(\pi_{1}\right) \\
& p_{2,1}=\varepsilon_{m+1} \circ \cdots \circ \varepsilon_{3}\left(\pi_{2} \varepsilon_{2}\left(\pi_{1}\right)\right) \\
& \vdots \\
& p_{m, 1}=\varepsilon_{m+1}\left(\pi_{m} \varepsilon_{m}\left(\cdots \varepsilon_{2}\left(\pi_{1}\right) \ldots\right)\right) \\
& q_{m+1,1}=\pi_{m+1} \varepsilon_{m+1}\left(\pi_{m} \varepsilon_{m}\left(\cdots \varepsilon_{2}\left(\pi_{1}\right) \ldots\right)\right)
\end{aligned}
$$

are factors of both members of $\lambda$ and represent $m+1$ elements of $\bar{\Omega}_{X_{m+1}}$ S. Hence, for each map $\varphi: X_{m+1} \longrightarrow S$, at least two of these expressions have the same image under the mapping $\bar{\varphi}: \bar{\Omega}_{X_{m+1}} \mathbf{S} \longrightarrow S$. For a fixed $\varphi$, suppose that these two expressions have indices $i$ and $j$ and that $i>j$. In case $m+1>i>j \geq 1$, then $\bar{\varphi}\left(p_{i, 1}\right)=\bar{\varphi}\left(p_{j, 1}\right)$, otherwise, $m+1=i>j \geq 1$ and $\bar{\varphi}\left(q_{m+1,1}\right)=\bar{\varphi}\left(p_{j, 1}\right)$. Consider the case where $m+1>i>j \geq 1$ (the proof is similar if $m+1=i>j \geq 1$ ). Then, for $l \geq 1, \bar{\varphi}\left(p_{i, j+1}\right)^{l} \cdot \bar{\varphi}\left(p_{j, 1}\right)=\bar{\varphi}\left(p_{j, 1}\right)$. Consequently, applying $\bar{\varphi}$ to both members of $\lambda$, we conclude that

$$
\begin{gathered}
\bar{\varphi}\left(q_{m+1,0}^{u}\right)=\bar{\varphi}\left(q_{m+1,0}^{v}\right) \\
\text { if and only if } \\
\bar{\varphi}\left(q_{m+1, i+1}\left(p_{i, j+1}\right)^{l} p_{j, 0}^{u}\right)=\bar{\varphi}\left(q_{m+1, i+1}\left(p_{i, j+1}\right)^{l} p_{j, 0}^{v}\right) .
\end{gathered}
$$


Since $\lambda$ belongs to a basis of pseudoidentities of $\mathbf{V}^{m+2}\left(B_{2}\right)$, then the pseudoidentity

$$
\varrho_{1}=\left(q_{i, 0}^{u}=q_{i, 0}^{v}\right)
$$

holds in $\mathbf{V}^{i+1}\left(B_{2}\right)$ and, by theorem $2.3, g \mathbf{V}^{i+1}\left(B_{2}\right) \models\left(\varrho_{1}, \mathcal{A}_{\pi_{i}}\right)$. Let

$$
\begin{aligned}
& X_{i+1}^{\prime}=X_{i}, \\
& \varepsilon_{i+1}^{\prime}: \quad \bar{\Omega}_{\mathcal{A}_{i}} \mathbf{S d} \longrightarrow \bar{\Omega}_{X_{i+1}^{\prime}} \mathbf{S} \\
& x \in E\left(\mathcal{A}_{\pi_{i}}\right) \longmapsto x, \\
& \pi_{i+1}^{\prime}=q_{i, j+1} .
\end{aligned}
$$

The labelings of $\mathcal{A}_{\pi_{i}}$ defined by $\pi_{i+1}^{\prime}$ and $\varepsilon_{i+1}^{\prime}$ are compatible with $\mathbf{V}\left(B_{2}\right)$ if and only if

$$
B_{2} \models \pi_{i+1}^{\prime} \varepsilon_{i+1}^{\prime}\left(w_{q}\right)=\pi_{i+1}^{\prime} \varepsilon_{i+1}^{\prime}\left(w_{q}^{\prime}\right),
$$

for $q \in V\left(\mathcal{A}_{\pi_{i}}\right)$ and $w_{q}, w_{q}^{\prime} \in \mathcal{A}_{\pi_{i}}^{*}\left(i\left(\pi_{i}\right) \delta_{\pi_{i}}, q\right)$, which we may rewrite as $B_{2}=q_{i, j+1} w_{q}=q_{i, j+1} w_{q}^{\prime}$. Note that $q_{i, j+1}, w_{q}, w_{q}^{\prime} \in E\left(\bar{\Omega}_{\mathcal{A}_{i}} \mathbf{S d}\right)$ and $i\left(w_{q}\right) \delta_{\pi_{i}} i\left(w_{q}^{\prime}\right)$ so, the set of pairs

$$
\left\{\left(t\left(q_{i, j+1}\right)^{-1}, i\left(w_{q}\right)\right)\right\} \cup \delta_{\pi_{i}}
$$

generate the same equivalence relation as the set

$$
\left\{\left(t\left(q_{i, j+1}\right)^{-1}, i\left(w_{q}^{\prime}\right)\right)\right\} \cup \delta_{\pi_{i}} .
$$

Hence, the compatibility conditions are satisfied and theorem 2.1 leads to the conclusion that the pseudoidentity

$$
\varrho_{2}=\left(\left(q_{i, j+1}\right)^{2} \cdot \varepsilon_{i} \circ \cdots \circ \varepsilon_{j+1}\left(q_{j, 0}^{u}\right)=\left(q_{i, j+1}\right)^{2} \cdot \varepsilon_{i} \circ \cdots \circ \varepsilon_{j+1}\left(q_{j, 0}^{v}\right)\right)
$$

holds in $\mathbf{V}^{i+2}\left(B_{2}\right)$, and, by theorem $2.3, g \mathbf{V}^{i+2}\left(B_{2}\right) \models\left(\varrho_{2}, \mathcal{A}_{\pi_{i+1}^{\prime}}\right)$ (note that $\mathcal{A}_{\pi_{i+1}^{\prime}}=\mathcal{A}_{\pi_{i}}$ ).

Using the same arguments, by theorems 2.3 and 2.1, making $k-(m+2)$ times the choice of the labelings as in $(3)$, we conclude that the following pseudoidentity, denoted $\varrho_{k-(m+1)}$,

$\left(q_{i, j+1}\right)^{k-(m+1)} \cdot \varepsilon_{i} \circ \cdots \circ \varepsilon_{j+1}\left(q_{j, 0}^{u}\right)=\left(q_{i, j+1}\right)^{k-(m+1)} \cdot \varepsilon_{i} \circ \cdots \circ \varepsilon_{j+1}\left(q_{j, 0}^{v}\right)$

holds in $\mathbf{V}^{i+k-(m+1)}$. Consequently,

$$
g \mathbf{V}^{i+k-(m+1)}\left(B_{2}\right) \models\left(\varrho_{k-(m+1)}, \mathcal{A}_{\pi_{i}}\right) .
$$

Now, for $t=1, \ldots,(m+1-i)$, making the choices:

$$
\begin{aligned}
& X_{i+k-(m+1)+t}^{\prime}=X_{i+t} \\
& \pi_{i+k-(m+1)+t}^{\prime}=\pi_{i+t} \\
& \varepsilon_{i+k-(m+1)+t}^{\prime}=\varepsilon_{i+t}
\end{aligned}
$$


we obtain labelings of $\mathcal{A}_{\pi_{i+k-(m+2)+t-1}^{\prime}}\left(=\mathcal{A}_{\pi_{i+t-1}}\right)$ compatible with $\mathbf{V}\left(B_{2}\right)$, since the pseudoidentity $\lambda$ holds in $\mathbf{V}^{m+2}\left(B_{2}\right)$. Hence

$$
q_{m+1, i+1} \cdot\left(p_{i, j+1}\right)^{k-(m+1)} \cdot p_{j, 0}^{u}=q_{m+1, i+1} \cdot\left(p_{i, j+1}\right)^{k-(m+1)} \cdot p_{j, 0}^{v}
$$

holds in $\mathbf{V}^{k}\left(B_{2}\right)$. Since $S \in \mathbf{V}^{k}\left(B_{2}\right)$, this pseudoidentity holds in $S$ and the equivalence (2) leads to the conclusion that $\bar{\varphi}\left(q_{m+1,0}^{u}\right)=\bar{\varphi}\left(q_{m+2,0}^{v}\right)$. So, $S$ satisfies $\lambda$.

Finally we can say that $S \in \mathbf{V}^{m+2}\left(B_{2}\right)$, because $S$ satisfies all pseudoidentities of the basis of $\mathbf{V}^{m+2}\left(B_{2}\right)$.

Theorem 4.2. The pseudovariety of semigroups $\mathbf{V}^{*}\left(B_{2}\right)$ is decidable.

\section{Proof}

The pseudovariety $\mathbf{V}\left(B_{2}\right)$ is decidable, because it is finitely generated [1]. In fact, for $X$ a finite set, $\bar{\Omega}_{X} \mathbf{V}\left(B_{2}\right)$ is finite and computable (these two conditions are equivalent to saying that $\mathbf{V}\left(B_{2}\right)$ is ordercomputable, property that implies decidability [2]).

By [1, theorem 10.2.3], there is a continuous embedding from $\bar{\Omega}_{X} \mathbf{V}^{n+1}\left(B_{2}\right)$ into $\bar{\Omega}_{Y} \mathbf{V}^{n}\left(B_{2}\right) * \Omega_{X} \mathbf{V}\left(B_{2}\right)$, for every $n \geq 1$, where $Y=X \times\left(\Omega_{X} \mathbf{V}\left(B_{2}\right)\right)^{1}$. Hence, if $\bar{\Omega}_{Y} \mathbf{V}^{n}\left(B_{2}\right)$ and $\Omega_{X} \mathbf{V}\left(B_{2}\right)$ are finite and computable then $\bar{\Omega}_{X} \mathbf{V}^{n+1}\left(B_{2}\right)$ is finite and computable.

By induction on $n$, we conclude that for every $n \geq 1$, the free finitely generated semigroups of $\mathbf{V}^{n}\left(B_{2}\right)$ are finite and computable. Hence, $\mathbf{V}^{n}\left(B_{2}\right)$ is decidable, for every $n \geq 1$.

Given a semigroup $S$, by theorem 4.1, $S \in \mathbf{V}^{*}\left(B_{2}\right)$ if and only if $S \in \mathbf{V}^{m}\left(B_{2}\right)$, where $m=\sharp(S)+2$, and, since $\mathbf{V}^{m}\left(B_{2}\right)$ is decidable, we conclude that it is decidable if $S$ is or is not an element of $\mathbf{V}^{*}\left(B_{2}\right)$.

\section{ANOTHER ALGORITHM TO SOLVE THE MEMBERSHIP PROBLEM FOR V* $\left(B_{2}\right)$}

In order to establish effective conditions to verify the membership relation to $\mathbf{V}^{*}\left(B_{2}\right)$, as an alternative to theorem 4.1 , we follow the suggestions given by J. Almeida in [1, section 10.10] to obtain a proof of decidability of $\mathbf{V}^{*}\left(B_{2}\right)$. Given a semigroup $S \in \mathbf{E R} \cap \mathbf{L R}$ a decomposition of $S$ based on a theorem of P. Stiffler [12] was suggested, and the problem becomes to test membership in $\mathbf{V}^{*}\left(B_{2}\right)$ of some factors of $S$.

Given a finite semigroup $S, I d_{m}(S)$ represents the set of its [0]minimal ideals and, for $s \in S, J_{s}$ represents the equivalence class of $s$ 
for the Green relation $\mathcal{J}$. So $S$ is a subdirect product of the semigroups

$$
S /\left\{s \in S: J_{s} \not I_{j} \backslash\{0\}, I_{j} \in I d_{m}(S)\right\}
$$

which are homomorphic images of $S$ and have a unique [0]-minimal ideal. Hence, $S \in \mathbf{V}$ if and only if all these factors belong to $\mathbf{V}$. Therefore, in the study of the membership relation to a pseudovariety, we may assume that $S$ has a unique [0]-minimal ideal, $I$. The semigroup $I$ is completely [0]-simple or nilpotent and $I \cong \mathcal{M}^{[0]}(G, R, C, P)$, a Rees matrix semigroup, where $P$ is a regular matrix or a null matrix, respectively. The set $C$ may be identified with the set of $\mathcal{L}$-classes (equivalence classes for the Green relation $\mathcal{L}$ ) of $I$, which do not contain the zero element, if it exists. We represent by $C^{*}$ the set of all $\mathcal{L}$-classes of $I$. Consider the right action of $S$ on $C^{*}$ defined by the following morphism from $S$ to the semigroup $\mathcal{T}_{C^{*}}$ of all functions $C^{*} \longrightarrow C^{*}$

$$
\begin{aligned}
\vartheta_{S}: S & \longrightarrow \mathcal{T}_{C^{*}} \\
s & \longmapsto t_{s}
\end{aligned} \text { where } \begin{aligned}
t_{s}: C^{*} & \longrightarrow C^{*} \\
L & \longmapsto L s=\{l s: l \in L\} .
\end{aligned}
$$

The subsemigroup $\vartheta_{S}(S)$ of $\mathcal{T}_{C^{*}}$ is represented by $R L M(S)$.

The following theorem has been proved by P. Stiffler and establishes a decomposition of a semigroup in terms of semidirect product. For $S$ and $T$ semigroups, by $S \odot T$ we denote the wreath product of $S$ by $T$ which is the semidirect product of $S^{\left(T^{1}\right)}$ by $T$.

Theorem 5.1. [12, theorem 3.1] With the above notation, let $S$ be a finite semigroup with a unique [0]-minimal ideal $I$ such that $I \cong$ $\mathcal{M}^{[0]}(G, R, C, P)$. Then,

i. in case $I$ is regular or $\sharp C>1$,
(a) if $S \neq I$,
$S \prec G \odot M \odot R L M(S) \odot S / I$,
(b) if $S=I$,$$
S \prec G \odot M \odot R L M(S) \odot U \text {; }
$$

ii. in case $\sharp C=1$,
(a) if $S \neq I$,
$S \prec G \odot M \odot S l_{2} \odot S / I$,
(b) if $S=I$,
$S \prec G \odot M \odot S l_{2} \odot U ;$

where $M$ is any monoid such that $\sharp M \geq \sharp R$, and $U$ is any of the semigroups $B(1,2)$, the right-zero semigroup with two elements, and $\mathrm{Sl}_{2}$, the semilattice with two elements.

If $S$ is aperiodic then the group $G$ is trivial and if we choose:

- $U=S l_{2}$;

- $M=\left\langle 1, x_{1}, \ldots, x_{r} \mid x^{2}=x, x y=y x, 1 x=x, x, y \in\left\{1, \ldots, x_{r}\right\}\right\rangle$ where $r=\sharp R$, 
then the factors of $S$ in Stiffler's decomposition are the semigroups $R L M(S)$ and $S / I$, and elements of Sl, the pseudovariety of all semilattices. Consequently, if $\mathbf{V}$ is a pseudovariety of aperiodic semigroups such that $\mathbf{S l} \subseteq \mathbf{V}$, then, $S \in \mathbf{V}^{*}$ if and only if $S / I$ and $R L M(S)$ are elements of $\mathbf{V}^{*}$.

The arguments we are going to develop involve induction on $\sharp S$. Note that, for a finite non trivial semigroup $S$ with a unique [0]-minimal $I, \sharp(S / I)<\sharp S$ and $\sharp R L M(S) \leq \sharp S$. If $\sharp R L M(S)=\sharp S$, then $R L M(S) \cong S$. So, the process of successive decompositions is finite and each terminal semigroup $T$ satisfies, at least, one of the following conditions: $T$ is trivial, $T$ satisfies the conditions of theorem 5.1 case (iib),or $T \cong R L M(T)$.

The structure of the semigroups $T$ such that $T \cong R L M(T)$ was studied in [1, section 10.10] and the result is presented in the next proposition.

Proposition 5.2. Let $S \in \mathbf{E R} \cap \mathbf{L R}$ with a unique [0]-minimal ideal I. With the above notation, if $S \cong R L M(S)$ then $I \cong B_{\sharp C}$ and $S / I$ is nilpotent.

Results of section 3 allow us to improve proposition 5.2.

Proposition 5.3. Let $S \in \mathbf{E R} \cap \mathbf{L R}$ be a semigroup with a unique [0]-minimal ideal $I$ and $S \cong R L M(S)$. Then $S \in \mathbf{V}^{*}\left(B_{2}\right)$ if and only if $S=I$.

\section{Proof}

If $S=I$ then, by proposition $5.2, S \cong B_{n}$ where $n+1$ is the number of $\mathcal{L}$-classes of $I$. Since $B_{n} \in \mathbf{V}\left(B_{2}\right)$, then $S \in \mathbf{V}^{*}\left(B_{2}\right)$.

If $S \neq I$ then there is $\sigma \in S \backslash I$. Each $s \in S$ induces a partial transformation on $C$, by restriction of $t_{s}$ to $C$, that is injective. Let us represent the corresponding morphism as $\vartheta_{S}^{\prime}: S \longrightarrow \mathcal{I}_{C}$, where $\mathcal{I}_{C}$ is the semigroup of partial injective transformations on $C$. Since $\vartheta_{S}$ is injective and 0 is a fixed point for transformations of $R L M(S)$, $S \cong R L M(S) \cong \vartheta_{S}^{\prime}(S)$. For $i=1, \ldots, n$, let $L_{i}$ be the $\mathcal{L}$-classes of $I$ which are elements of $C$. The non null elements of $\vartheta_{S}^{\prime}(I)$ are the partial injective transformations, represented by $s_{p, q}$, whose domain is a singular set, $\left\{L_{p}\right\}$, and whose image set is $\left\{L_{q}\right\}$, respectively, where $p, q \in\{1, \ldots, n\}$. By 0 we represent the empty transformation. So the domain of the partial injective transformation $\sigma^{\prime}=\vartheta_{S}^{\prime}(\sigma)$ has two or more elements, which means that $\sigma^{\prime}$ transforms $L_{i}$ in $L_{j}$ and $L_{k}$ in $L_{l}$, for some $i, j, k, l \in\{1, \ldots, n\}$ such that $i \neq k$. 
Consider the pseudoidentity

$$
(z x y x t z)^{\omega} x(y x)^{2}=(z x y x t z)^{\omega} x y x
$$

which holds in $\mathbf{V}^{*}\left(B_{2}\right)$ (as has been proved at the end of section 3 ) and the morphism $\bar{\psi}: \bar{\Omega}_{\{x, y, z, t\}} \mathbf{S} \longrightarrow \vartheta_{S}^{\prime}(S)$, continuous extension of $\psi:\{x, y, z, t\} \longrightarrow \vartheta_{S}^{\prime}(S)$ which is defined by

$$
\psi(x)=\sigma^{\prime}, \quad \psi(y)=s_{j, k}, \quad \psi(z)=s_{i, i}, \quad \psi(t)=s_{l, i} .
$$

Consequently, $\quad \bar{\psi}\left((z x y x t z)^{\omega}\right)=s_{i, i}$,

$$
\begin{aligned}
& \bar{\psi}\left(x(y x)^{2}\right)=0, \\
& \bar{\psi}(x y x)=s_{i, l},
\end{aligned}
$$

and $\bar{\psi}\left((z x y x t z)^{\omega} x(y x)^{2}\right) \neq \bar{\psi}\left((z x y x t z)^{\omega} x y x\right)$, which means that $S \notin \mathbf{V}^{*}\left(B_{2}\right)$.

Consequently, we obtain another proof that $\mathbf{V}^{*}\left(B_{2}\right) \neq \mathbf{E R} \cap \mathbf{L R}$ (see [13]) since there are semigroups $S$ in the conditions of the last proposition and such that $S \neq I$. As an example, consider $S$ the subsemigroup of $\mathcal{T}_{\{0,1,2,3,4,5\}}$ generated by the transformations

$$
\left(\begin{array}{lllll}
0 & 1 & 2 & 3 & 4 \\
0 & 1 & 3 & 0 & 0
\end{array}\right) \text { and }\left(\begin{array}{ccccc}
0 & 1 & 2 & 3 & 4 \\
0 & 2 & 0 & 4 & 1
\end{array}\right)
$$

Now we can write an algorithm to test if a member of $\mathbf{E R} \cap \mathbf{L R}$ belongs to $\mathbf{V}^{*}\left(B_{2}\right)$ :

\section{Algorithm 1}

ENTRY: $S \in \mathbf{E R} \cap \mathbf{L R}$

1.- If $\sharp S=1$ then $S \in \mathbf{V}^{*}\left(B_{2}\right)$.

2.- If $\sharp S>1$ then:

2.1- let $F=\left\{I_{j}: I_{j} \in I d_{m}(S)\right\}$;

2.2- for each $I_{j} \in F$ :

$$
\text { 2.2.1- } S_{j}=S /\left\{s \in S: J_{s} \nsupseteq I_{j} \backslash\{0\}\right\}
$$

2.2.2- if $R L M\left(S_{j}\right) \cong S_{j}$ and $S_{j}=I_{j}$ then $S_{j} \in \mathbf{V}^{*}\left(B_{2}\right)$;

2.2.3- if $R L M\left(S_{j}\right) \cong S_{j}$ and $S_{j} \neq I_{j}$ then $S_{j} \notin \mathbf{V}^{*}\left(B_{2}\right)$;

2.2.4- if $R L M\left(S_{j}\right) \not S_{j}$ then,

$$
\begin{aligned}
& \text { if } R L M\left(S_{j}\right), S_{j} / I_{j} \in \mathbf{V}^{*}\left(B_{2}\right) \\
& \text { then } S_{j} \in \mathbf{V}^{*}\left(B_{2}\right) \text {; } \\
& \text { else } S_{j} \notin \mathbf{V}^{*}\left(B_{2}\right) \text {; }
\end{aligned}
$$

2.3- if, for $j=1, \ldots, \sharp F, S_{j} \in \mathbf{V}^{*}\left(B_{2}\right)$

then $S \in \mathbf{V}^{*}\left(B_{2}\right)$

else $S \notin \mathbf{V}^{*}\left(B_{2}\right)$. 
Further considerations on the factors of $S$ in Stiffler's decomposition are stated in the following results, which lead to an improved algorithm.

Proposition 5.4. If $S \in \mathbf{E R} \cap \mathbf{L R}$ is a regular semigroup then $S \in \mathbf{V}^{*}\left(B_{2}\right)$.

\section{Proof}

The factors of $S$ in the subdirect product, that is the semigroups

$$
S_{j}=S /\left\{s \in S: J_{s} \nsupseteq I_{j} \backslash\{0\}, I_{j} \in I d_{m}(S)\right\}
$$

are regular and each has only one [0]-minimal ideal. By proposition 5.2, each one of those semigroups $S_{j}$ verifies either $S_{j} \neq R L M\left(S_{j}\right)$ or $S_{j}$ is equal to its 0-minimal ideal and so $S_{j} \in \mathbf{V}^{*}\left(B_{2}\right)$.

Suppose $S_{j} \neq R L M\left(S_{j}\right)$, for some index $j$. The semigroups $S_{j} / I_{j}$ and $R L M\left(S_{j}\right)$ are again regular semigroups of ER $\cap \mathbf{L R}$ and consequently, for each one, we may initialize a new decomposition.

Since $S$ is finite, this process finishes after a finite number of steps, and the terminal semigroups belong to $\mathbf{V}^{*}\left(B_{2}\right)$, because they are trivial semigroups, or satisfy conditions of theorem 5.1 case (iib), or are equal to the 0 -minimal ideal which is of the form $B_{n}$, by proposition 5.2. The final conclusion is that $S \in \mathbf{V}^{*}\left(B_{2}\right)$.

Corollary 5.5. Let $S \in \mathbf{E R} \cap \mathbf{L R}$ be a semigroup equal to its unique [0]-minimal ideal. Then $S \in \mathbf{V}^{*}\left(B_{2}\right)$.

\section{Proof}

Under the hypotheses, $S$ is a completely [0]-simple semigroup, and so is regular, or $S$ is isomorphic to a nilpotent semigroup with two elements. So, in both cases, $S \in \mathbf{V}^{*}\left(B_{2}\right)$.

Lemma 5.6. Let $S \in \mathbf{E R}$ be a semigroup with only one [0]-minimal ideal $I$, which is regular. Then the semigroup $R L M(S)$ has only one [0]-minimal ideal, which is an aperiodic Brandt semigroup.

\section{Proof}

The following proof contains arguments similar to the arguments used by J. Almeida to obtain the result stated in lemma 5.2.

If $S$ does not have a zero and since $I \in \mathbf{E R}$, then $I$ has only one $\mathcal{L}$-class and $R L M(S)$ is trivial. So, suppose $S$ is a not trivial semigroup which has a zero. Hence, $\operatorname{RLM}(S)$ is non trivial and is the homomorphic image of $S$ under $\vartheta_{S}$. Consequently, $\vartheta_{S}(I)$ is regular and is the unique 0-minimal ideal of $\operatorname{RLM}(S)$.

Let $e, f \in I$ be two idempotents. If $e \mathcal{L} f$ then $\vartheta_{S}(e)=\vartheta_{S}(f)$, else $\vartheta_{S}(e)$ and $\vartheta_{S}(f)$ are not $\mathcal{L}$-equivalent. So, $\vartheta_{S}(I)$ does not contain 
$\mathcal{L}$-equivalent idempotents and, since $\vartheta_{S}(I) \in \mathbf{E R}$, does not contain $\mathcal{R}$-equivalent idempotents. Consequently, the product of idempotents in $\vartheta_{S}(I)$ is commutative. Hence we conclude that $\vartheta_{S}(I)$ is a completely 0 -simple inverse semigroup, which means that it is a Brandt semigroup.

Moreover, since $I$ does not contain $\mathcal{R}$-equivalent idempotents, the elements of $\vartheta_{S}(I)$ are transformations such that only one element of the domain may have image not zero. So $\vartheta_{S}(I)$ is aperiodic

The semigroup $R L M(S) / \vartheta_{S}(I)$ is the image of $S / I$ by a morphism $\vartheta$ such that $p_{\vartheta_{S}(I)} \circ \vartheta_{S}=\vartheta \circ p_{I}$, where $p_{\vartheta_{S}(I)}$ is the projection of $R L M(S)$ in $R L M(S) / \vartheta_{S}(I)$ and $p_{I}$ is the projection of $S$ in $S / I$. If $S$ satisfies lemma 5.6's conditions, then $R L M(S)$ satisfies them too, and so $R L M(R L M(S))$ has only one 0-minimal ideal, which is a Brandt semigroup isomorphic to the 0-minimal ideal of $R L M(S)$, as we can deduce from the proof of lemma 5.6. Consequently, $R L M(R L M(R L M(S)))$ is isomorphic to $R L M(R L M(S))$.

If $S$ has only one [0]-minimal ideal $I$ which is non regular, then $I$ is nilpotent, $\vartheta_{S}(I)=0$ and $R L M(S)$ is an image of $S / I$. In this case, $R L M(S)$ can have several 0 -minimal ideals and $\sharp R L M(S)<\sharp S$.

Given $S \in \mathbf{E R} \cap \mathbf{L R}$ with $r \mathcal{J}$-classes that do not contain 0, $\left(J_{i}\right)_{i \in\{1, \ldots, r\}}$, to execute the algorithm 1 is equivalent to test if each semigroup of the family

$$
\mathcal{F}=\left(S /\left\{a \in S: J_{a} \nsupseteq J_{i}\right\}\right)_{i \in\{1, \ldots, r\}}
$$

is an element of $\mathbf{V}^{*}\left(B_{2}\right)$ and, $S \in \mathbf{V}^{*}\left(B_{2}\right)$ if and only if $\mathbf{V}^{*}\left(B_{2}\right)$ contains $\mathcal{F}$. Note that $\mathcal{F}$ is partially ordered by the order relation $\prec_{q}$ defined by: $T \prec_{q} D$ if and only if $T$ is a quotient of $D$. The minimum element of $\mathcal{F}$ is the trivial semigroup and there are as many maximal elements as 0-minimal ideals. If a semigroup covers the trivial semigroup then it is a completely [0]-simple semigroup or a nilpotent semigroup with two elements.

For each $S_{i} \in \mathcal{F}$, we represent its 0 -minimal ideal by $I_{i}$. So, $S_{i} / I_{i}$ is a subdirect product of $\mathcal{F}_{i}=\left(S_{i_{k}}\right)_{i_{k} \in K}$, where $K \subset\{1, \ldots, r\}$ and each $S_{i_{k}} \in \mathcal{F}_{i}$ satisfies $S_{i_{k}} \prec_{q} S_{i}$ and $S_{i_{k}} \neq S_{i}$. Hence, the criterion to test if $S_{i} \in \mathbf{V}^{*}\left(B_{2}\right)$ is the following:

1. if $S_{i}=I_{i}$ or $S_{i}$ is regular then $S_{i} \in \mathbf{V}^{*}\left(B_{2}\right)$;

2. else, if $S_{i} \cong R L M\left(S_{i}\right)$ then $S_{i} \notin \mathbf{V}^{*}\left(B_{2}\right)$;

3. otherwise, $S \in \mathbf{V}^{*}\left(B_{2}\right)$ if and only if $\mathcal{F}_{i} \cup\left\{R L M\left(S_{i}\right)\right\} \subseteq \mathbf{V}^{*}\left(B_{2}\right)$.

In case 3 and if $I_{i}$ is not regular then $\operatorname{RLM}\left(S_{i}\right)$ is a morphic image of $S_{i} / I_{i}$, then $R L M\left(S_{i}\right) \in \mathbf{V}^{*}\left(B_{2}\right)$ if and only if the semigroups $S_{j} \in \mathcal{F}$, such that $S_{j} \prec_{q}\left(S_{i} / I_{i}\right)$, are members of $\mathbf{V}^{*}\left(B_{2}\right)$. If $I_{i}$ is regular then, 
by proposition 5.6, $R L M\left(S_{i}\right)$ has only one 0 -minimal ideal, which is a Brandt semigroup and, $R L M\left(S_{i}\right) \in \mathbf{V}^{*}\left(B_{2}\right)$ if and only if the semigroups $S_{j} \in \mathcal{F}$, such that $S_{j} \prec_{q}\left(S_{i} / I_{i}\right)$, are members of $\mathbf{V}^{*}\left(B_{2}\right)$ and $R L M\left(R L M\left(S_{i}\right)\right)$ is completely 0-simple.

So we can write a new algorithm.

\section{Algorithm 2}

\section{ENTRY: $S \in \mathbf{E R} \cap \mathbf{L R}$}

1.- If either $S$ is regular or $S$ is nilpotent then $S \in \mathbf{V}^{*}\left(B_{2}\right)$.

2.- Else :

2.1- $J=\left\{J_{j}: J_{j}\right.$ non null $\mathcal{J}$-class of $\left.S\right\}$;

2.2- for each $j \in\{1, \ldots, \sharp J\}$

2.2.1- let $S_{j}=S /\left\{s \in S: J_{s} \nsupseteq J_{j}\right\}$;

2.2.2- if either $S_{j}$ is regular or, $\sharp S_{j}=2$ and $S_{j}$ is nilpotent, then $S_{j} \in \mathbf{V}^{*}\left(B_{2}\right)$;

2.2.3- else if $R L M\left(S_{j}\right) \cong S_{j}$ then $S_{j} \notin \mathbf{V}^{*}\left(B_{2}\right)$;

2.2.4- else if $R \operatorname{LM}\left(R \operatorname{RM}\left(S_{j}\right)\right)$ is equal to its 0-minimal ideal then $S_{j} \in \mathbf{V}^{*}\left(B_{2}\right)$; else $S_{j} \notin \mathbf{V}^{*}\left(B_{2}\right)$;

2.3- if, for some $1 \leq j \leq \sharp J, S_{j} \notin \mathbf{V}^{*}\left(B_{2}\right)$

then $S \notin \mathbf{V}^{*}\left(B_{2}\right)$;

else $S \in \mathbf{V}^{*}\left(B_{2}\right)$.

\section{END}

Let $S$ be a finite semigroup with $n$ elements. Testing if $S \in \mathbf{E R} \cap \mathbf{L R}$ is equivalent to testing if $S \models(\text { exeye })^{\omega} x=(\text { exeye })^{\omega}$, and this can be done by an algorithm of $O\left(n^{3}\right)$ using a table for the function that associates $s^{\omega}$ to each $s \in S$, which can be computed in polynomial time of $O\left(n^{2}\right)$. The execution of algorithm 2 is the result of the execution of a finite number of basic steps, which is bounded by a linear function on $\sharp J$. The basic steps are to test if a semigroup is regular or nilpotent, to compute the $\mathcal{J}$-classes of $S$, the Rees-quotients of the form $S_{j}$ and their homomorphic images $R L M\left(S_{j}\right)$ and $R L M\left(R L M\left(S_{j}\right)\right)$, and to test if $R L M\left(S_{j}\right) \cong S_{j}$. To verify if $S$ is regular or nilpotent we need to compute no more than $3 n^{2}$ products of elements of $S$. The calculation of the Green classes structure of $S$ takes $2 n^{2}$ products, since Green classes of $S$ are defined by comparison of sets of the form $a S^{1}$ and of sets of the form $S^{1} a$, for all $a \in S$. If we know the Green classes structure of $S$, the computation of semigroups $S_{j}$ and of their Green classes structure is trivial. Given a semigroup $T$, if we know its Green classes structure, to compute $R L M(T)$ takes less than $\sharp T^{2}$ products. 
So, the computation of the semigroups $R L M\left(S_{j}\right)$ and $R L M\left(R L M\left(S_{j}\right)\right)$ takes less than $2 n^{2}$ products. Testing if $\operatorname{RLM}\left(S_{j}\right) \cong S_{j}$ is equivalent to testing if $\sharp R L M\left(S_{j}\right)=\sharp S_{j}$. Consequently, we have an algorithm of polynomial complexity of $O\left(n^{3}\right)$ to test if a finite semigroup $S$ with $n$ elements belongs to $\mathbf{V}^{*}\left(B_{2}\right)$.

\section{ACKNowledgements}

I would like to thank Jorge Ameida and Assis Azevedo for reading the previous version and for their useful comments.

\section{REFERENCES}

[1] J. Almeida. Finite semigroups and Universal Algebra, World Scientific, Singapore, 1995. Original version in Portuguese, 1992.

[2] J. Almeida. Some algoritmic problems for pseudovarieties. Technical report, (9/96), 1996.

[3] J. Almeida. A syntactical proof of locality of DA. Int. J. Algebra and Computation, 6(1996) 165-177.

[4] J. Almeida, A. Azevedo, and L. Teixeira. On finitely based pseudovarieties of the form $V * D$ and $V * D_{n}$. J. Pure Appl. Algebra, 146(2000) 1-15.

[5] J. Almeida and P. Weil. Relatively free profinite monoids. Semigroups, formal languages and groups, J. Fountain ed., Série C. Mathematical and Physical Sciencies, 466(1995) 73-118.

[6] J. Almeida and P. Weil. Profinite categories and semidirect products. J. Pure Appl. Algebra, 123(1998) 1-50.

[7] S. Eilenberg. Automata, languages and machines, volume B of Pure and Applied Mathematics. Academic Press, New York, 1976.

[8] J.-E. Pin. Varieties of formal languages. Plenum Press, 1986.

[9] N. Reilly. Free combinatorial strict inverse semigroups. J. London Mathematical Society, 39(2)(1989) 102-120.

[10] J. Reiterman. The Birkhoff theorem for finite algebras. Algebra Universalis, 14(1982) 1-10.

[11] J. Rhodes. Undecidability, automata and pseudovarieties of finite semigroups. Technical report, 1997.

[12] P. Stiffler. Extension of the fundamental theorem of finite semigroups. $A d$ vances in Math., 11(1973) 159-209.

[13] L. Teixeira. On semidirectly closed pseudovarieties of aperiodic semigroups. $J$. Pure Appl. Algebra, to appear.

[14] B. Tilson. Categories as algebras: an essential ingredient in the theory of monoids. J. Pure Appl. Algebra, 48(1987) 83-198.

[15] A. Trahtman. A basis of identities of the five element Brandt semigroups. Ural Gos.Univ.Mat.Zap., 12(1981) 147-149.

Departamento de Matemática, Universidade do Minho, Campus de Gualtar, 4700-320 Braga, Portugal

E-mail address: mlurdes@math.uminho.pt 